\section{Local dornase alfa treatment reduces NETs-induced airway obstruction during severe RSV infection}

\section{ABSTRACT}

Respiratory syncytial virus (RSV) infection is characterised by airway obstruction with mucus plugs, containing DNA networks in the form of neutrophil extracellular traps (NETs). We investigated the effect of dornase alfa on histopathological NETs-induced airway obstruction and viral load in an age-relevant calf model of severe bovine RSV disease. As compared with the control animals, dornase alfa treatment resulted in a strong reduction of NETs-induced airway obstruction. Viral load in the lower respiratory tract was not different between the two groups. We conclude that NETs form a relevant target for treatment of airway obstruction in severe RSV disease.

\section{INTRODUCTION}

Respiratory syncytial virus (RSV) is among the most important respiratory pathogens in children worldwide. ${ }^{1}$ Small airway obstruction by dense plugs, composed of mucus and cellular debris from neutrophils and sloughed bronchial epithelial cells, is a key histopathological feature in children with severe RSV-lower respiratory tract disease (LRTD). ${ }^{2}$ Recently, we have implicated neutrophil extracellular traps (NETs) in the development of airway obstruction in severe RSV-LRTD. ${ }^{3}$

NETs are networks of extracellular DNA covered with antimicrobial peptides and histones, which contribute to the capture and inactivation of bacteria, fungi and viruses, including RSV. ${ }^{4}$ However, there appears a delicate balance between aid and damage to the host as accumulating evidence now suggests that NETs can cause lung injury and may contribute to airway obstruction by trapping mucus into large, viscous plugs. ${ }^{4}$ As such, pharmacological targeting of NETs, for example, by degradation of the DNA backbone by dornase alfa, may be of therapeutic benefit.

In this study, we hypothesised that local dornase alfa treatment reduces NETs-induced airway obstruction in the airways during experimental severe RSV-LRTD. To test this hypothesis, we used the well-established cognate host-virus model of bovine RSV (bRSV) infection in calves, which is characterised by neutrophilic inflammation with NETs formation and histopathological evidence of (small) airway obstruction, similar to severe RSV-LRTD in children. ${ }^{5-7}$

\section{METHODS}

Twelve 4-week-old, colostrum-deprived dairy calves were infected on day 0 with $3.6 \log _{10}$ TCID 50 of bRSV (Odijk strain, seventh in vivo passage) in a $2 \mathrm{~mL}$ volume. Next, the calves (six per group) received
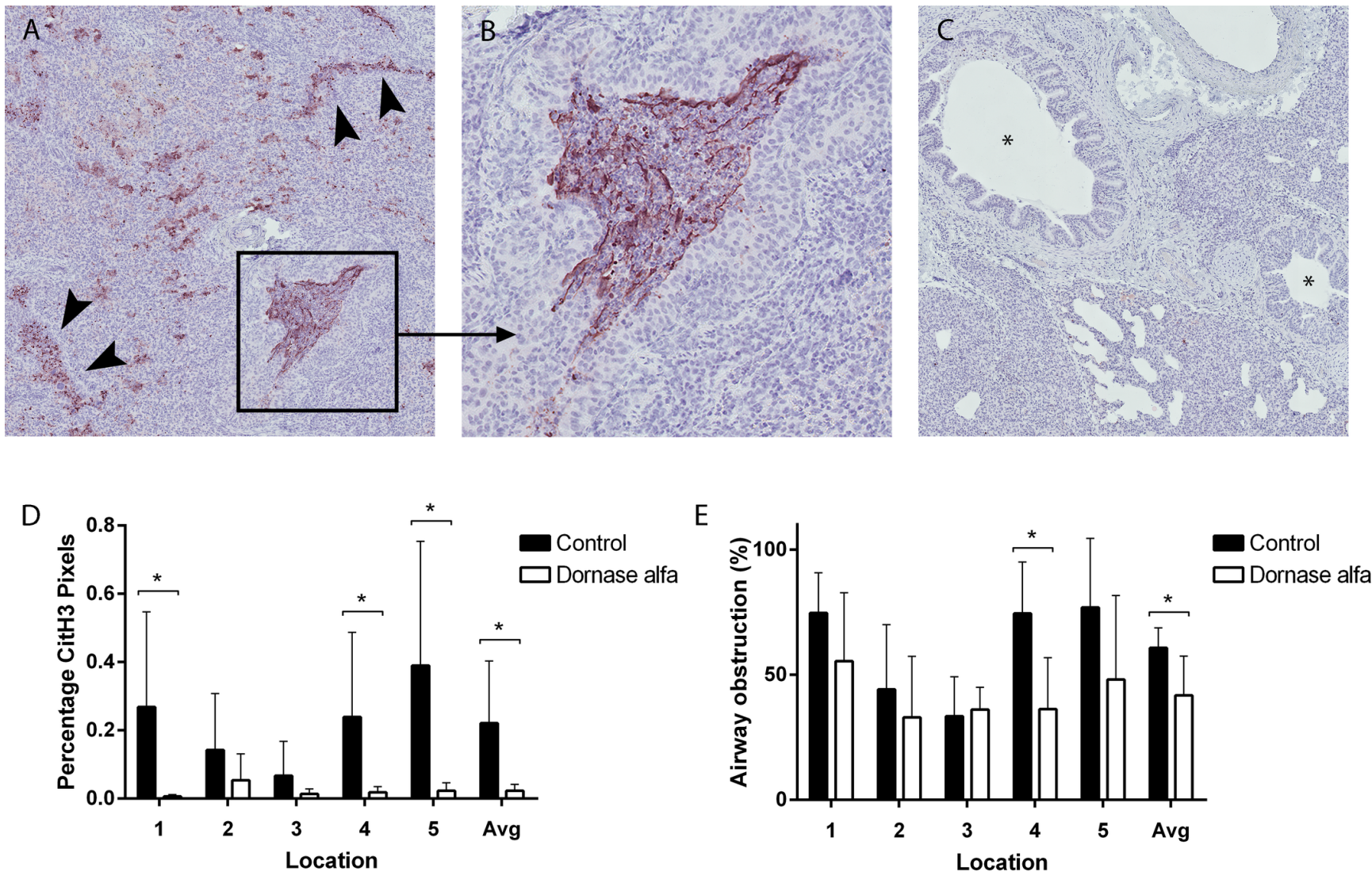

Figure 1 Neutrophil extracellular traps (NETs) degradation by dornase alfa treatment. Immunohistochemistry of lung tissue sections, stained for citrullinated histone $\mathrm{H3}$ (CitH3) to detect NETs formation. (A) Severe airway obstruction by NETs-rich plugs (black arrowheads and black square) in a saline (control)-treated calf (representative image, magnification 100x), with (B) magnification (450x) of the square. (C) Open airways (asterisks) with absence of NETs-rich obstructing plugs in a dornase alfa-treated calf (representative image, magnification 100x). (D) Percentages of intraluminal airway CitH3-positive pixels for the five different lung locations (see online supplementary figure 1 ) in control calves ( $n=6$, black bars) and dornase alfa-treated calves ( $n=6$, white bars), average (Avg) of the five locations: $p=0.04$. (E) Percentages of partially and completely obstructed airways counted in whole lung tissue sections for the five different locations (see online supplementary figure 2 ) in control calves ( $n=6$, black bars) compared with dornase alfa-treated calves ( $n=6$, white bars), average (Avg) of the five locations: $p=0.03$, location 4: $p=0.03$. 


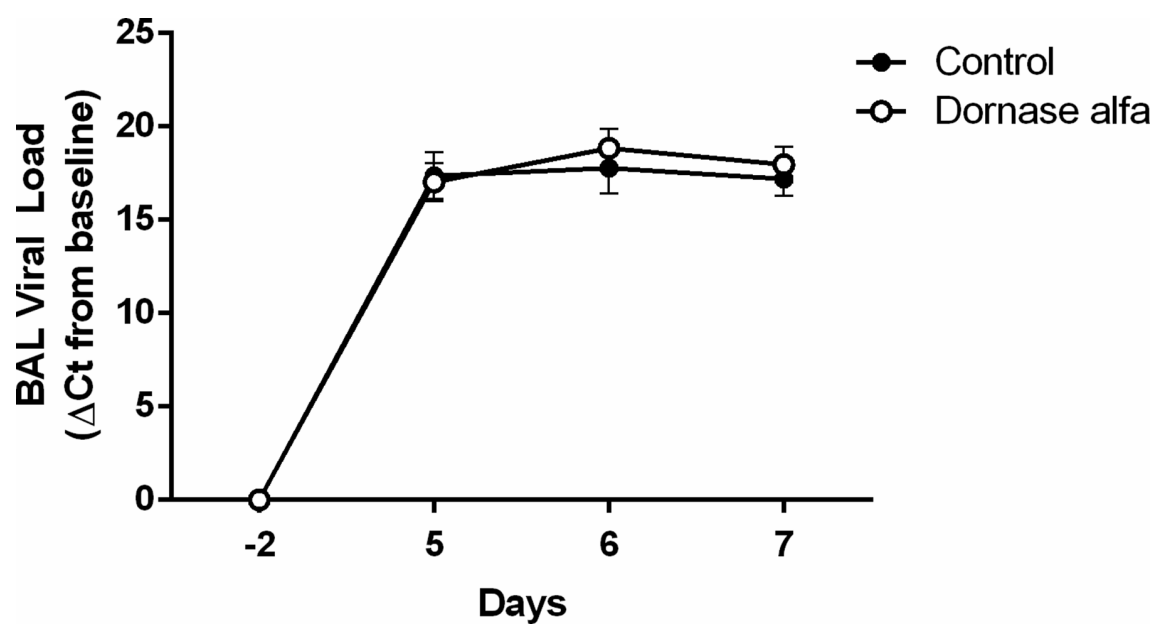

Figure 2 Lung viral load in bronchoalveolar lavage (BAL) from control (black circles) and dornase alfa-treated (white circles) calves during bovine respiratory syncytial virus (bRSV) lower respiratory tract disease, as detected by reverse transcription-PCR. Data are expressed as mean $\pm \mathrm{SD}$ of the $\triangle \mathrm{Ct}$ (total number PCR cycles minus threshold cycle). $n=6$ calves per group, $\mathrm{p}=0.1$ between groups.

either twice daily $5 \mathrm{~mL}$ dornase alfa (treatment group, Pulmozyme $1 \mathrm{mg} / \mathrm{mL}$, Hoffmann-La Roche, Basel, Switzerland) or $0.9 \% \mathrm{NaCl}$ (control group), starting on day 5 after viral inoculation. Further details can be found in the online supplementary methods.

\section{RESULTS}

Overall, there was a significant reduction of NETs in the dornase alfa-treated animals compared with the control group (figure $1 \mathrm{~A}-\mathrm{D}, \mathrm{p}=0.02$ ). On average, the percentage of obstructed airways in the dornase alfa group was 31\% lower compared with normal saline group $(41.8 \% \pm 6.4 \%$ vs $60.7 \pm 3.3 \%$, respectively, figure $1 \mathrm{E}, \mathrm{p}=0.03)$, with a highest improvement of $51 \%$ in the left cranial lung area $(36.3 \% \pm 8.4 \%$ vs $74.5 \pm 8.4 \%$, figure $1 \mathrm{E}, \mathrm{p}=0.03)$. There was no difference in the viral loads between the calves treated with dornase alfa versus normal saline during any of the treatment days (figure 2). There were no differences in the lung inflammatory response as determined by total and differential bronchoalveolar lavage (BAL) cells between the animals treated with dornase alfa or normal saline (see online supplementary results). The calves treated with dornase alfa had a trend towards lower LRTD scores $(p=0.07)$ and had reduced extent of hypercapnia on day 7 $(51.5 \pm 2.8 \mathrm{~mm} \mathrm{Hg}$ vs $61.7 \pm 4.7 \mathrm{~mm} \mathrm{Hg}$, $\mathrm{p}=0.04)$ compared with calves treated with normal saline (see online supplementary results). Interestingly, four out of the six animals in the control group had to be sacrificed prematurely due to reaching their humane end point based on acute deterioration, as opposed to two out of six calves in the dornase alfa group based on persistent severe disease (see online supplementary results). Further results on lung inflammation and clinical responses can be found in the online supplementary results.

\section{DISCUSSION}

Over the last years, NETs are increasingly being implicated in the pathophysiology of multiple respiratory diseases, including cystic fibrosis, COPD, acute lung injury and pulmonary infections. ${ }^{4}$ Specifically, the formation of NETs in the respiratory tract may contribute to airway obstruction, by deposition of large web-like networks with high DNA content, thereby increasing mucus viscosity. ${ }^{348}$ Previously, we have shown the formation of NETs in bronchoalveolar lavage fluid (BALF) from infants with severe RSV-LRTD, as well as in mucus plugs obstructing the airways of bRSV-infected calves. ${ }^{3}$ In the present study, we confirmed the hypothesis that NETs are actively involved in airway obstruction during severe bRSVLRTD. Local dornase alfa treatment strongly reduced the amount of NETs in the airways (figure 1A-D), which was associated with a reduction in airway occlusion on histopathological evaluation (figure 1E). Interestingly, the lysis of NETs led to increased DNA content in BALF, suggesting fragmentation and 'freeing' the DNA from disintegrated mucus plugs (see online supplementary results), similar to reports in patients with cystic fibrosis. ${ }^{9}$
On the other hand, disruption of the protective functions of NETs might also be disadvantageous. NETs are able to capture RSV particles in vitro, ${ }^{34}$ and as such, a theoretical concern may be renewed release and enhanced dissemination of immobilised virions on lysis of NETs. However, there was no increased viral dissemination (figure 2), nor evidence of enhanced direct viral-induced lung pathology or inflammation (see online supplementary results) in our in vivo bRSV calf model. These data suggest a relatively limited role of NETs in antiviral defence in the respiratory tract, but future studies must continue to evaluate these potential risks.

In our 'proof of principle' study focused on targeting NETs (see online supplementary limitations), we observed improvement of clinical indices, most prominently a relevant reduction in the extent of hypercapnia, on dornase alfa treatment (see online supplementary results). Although these findings need to be interpreted with care because our study was not specifically powered to detect robust clinical effects, this may form the basis of further testing of a potential clinical benefit of dornase alfa and other future interventions in NETs biology (eg, PAD4 inhibitors). The clinical use of nebulised dornase alfa treatment during mild to moderate RSV disease in infants has previously been investigated. ${ }^{10-12}$ While one prospective randomised trial showed a strong reduction in chest X-ray abnormalities, including atelectasis, ${ }^{11}$ no clinical benefit, and even a statistically non-significant prolonged hospitalisation, of the routine use of nebulised dornase alfa was demonstrated in two subsequent randomised placebo-controlled trials and meta-analysis. ${ }^{10} 1213$ However, in a case series in mechanically ventilated children with severe RSV-LRTD, a population that suffers much more from the typical airway mucus obstruction with atelectasis, dornase alfa nebulisation was of benefit with both clinical and radiological improvement, ${ }^{14}$ which is a finding in line with observations in daily clinical practice. Future studies powered for clinical effect must determine if targeting of NETs is of actual clinical benefit during severe RSV-LRTD.

In conclusion, local dornase alfa treatment strongly reduces the amount of NETs in the respiratory tract, leading to less airway obstruction in calves with bRSV-LRTD. These results suggest that NETs contribute to the pathogenesis of airway obstruction during severe RSV-LRTD. Targeting NETs formation 
could prove a promising new treatment to alleviate airway obstruction in severe RSV-LRTD.

\section{Bart Cortjens, ${ }^{1}$ Rineke de Jong, ${ }^{2}$ Judith G Bonsing, ${ }^{2}$ Job B M van Woensel, ${ }^{1}$ Adriaan F G Antonis, ${ }^{2}$ Reinout A Bem ${ }^{1}$}

$B C$ and RJ contributed equally.

'Paediatric Intensive Care, Emma Children's Hospital, Amsterdam, The Netherlands

${ }^{2}$ Wageningen University and Research, Bioveterinary Research, Lelystad, The Netherlands

Correspondence to Bart Cortjens, Paediatric Intensive Care, Emma Children's Hospital, Meibergdreef 9, Amsterdam 1100 DD, The Netherlands; b.cortjens@amc.nl

Acknowledgements The authors thank N StockhofeZurwieden and S Vreman, Wageningen Bioveterinary Research, Lelystad, The Netherlands, for expert lung pathology analysis.

Contributors $B C, J B M v W, R d J, A F A$ and RAB designed the study. BC, RdJ, JGB and AFA conceived and carried out the animal experiments. BC, RdJ and JGB carried out the BAL measurements and immunohistochemistry and analysed the data. BC, RdJ, AFA, JBMvW and RAB interpreted the results. All authors were involved in writing the paper and had final approval of the submitted and published versions.

Funding This work was supported by the Emma Children's Hospital Support Foundation [CC200001] and the Amsterdam Economic Board [EZ1311 ALOHA RSV].

Competing interests None declared.

Ethics approval This study was conducted under legislation ofthe Dutch Central Authority for Scientific procedures on Animals and after approved of the
Body of Animal Welfare of Wageningen University and Research.

Provenance and peer review Not commissioned; externally peer reviewed.

(c) Article author(s) (or their employer(s) unless otherwise stated in the text of the article) 2018. All rights reserved. No commercial use is permitted unless otherwise expressly granted.

- Additional material is published online only. To view please visit the journal online (http://dx.doi.org/ 10.1136/thoraxjnl-2017-210289)

\section{Check for updates}

To cite Cortjens B, de Jong R, Bonsing JG, et al. Thorax 2018:73:578-580

Received 21 March 2017

Revised 4 July 2017

Accepted 17 July 2017

Published Online First 5 August 2017

Thorax 2018:73:578-580

doi:10.1136/thoraxinl-2017-210289

\section{REFERENCES}

1 Meissner HC. Viral Bronchiolitis in Children. N Engl J Med 2016:374:62-72.

2 Pickles RJ, DeVincenzo JP. Respiratory syncytial virus (RSV) and its propensity for causing bronchiolitis. J Pathol 2015;235:266-76.

3 Cortjens B, de Boer OJ, de Jong R, et al. Neutrophil extracellular traps cause airway obstruction during respiratory syncytial virus disease. J Pathol 2016;238:401-11.

4 Cortjens B, van Woensel JB, Bem RA. Neutrophi extracellular traps in respiratory disease: guided anti-microbial traps or toxic webs? Paediatr Respir Rev 2017:21:54-61.

5 Bem RA, Domachowske JB, Rosenberg HF. Animal models of human respiratory syncytial virus disease. Am J Physiol Lung Cell Mol Physiol 2011;301:L148-56.

6 Cortjens B, de Boer OJ, de Jong R, et al. Neutrophil extracellular traps cause airway obstruction during respiratory syncytial virus disease. J Pathol 2016:238:401-11.

7 Everard ML, Swarbrick A, Wrightham M, et al. Analysis of cells obtained by bronchial lavage of infants with respiratory syncytial virus infection. Arch Dis Child 1994:71:428-32.

8 Shak S, Capon DJ, Hellmiss R, et al. Recombinant human DNase I reduces the viscosity of cystic fibrosis sputum. Proc Natl Acad SciU SA 1990:87:9188-92.

9 Shah PL, Scott SF, Knight RA, et al. In vivo effects of recombinant human DNase I on sputum in patients with cystic fibrosis. Thorax 1996;51:119-25.

10 Boogaard R, Hulsmann AR, van Veen L, et al. Recombinant human deoxyribonuclease in infants with respiratory syncytial virus bronchiolitis. Chest 2007;131:788-95.

11 Nasr SZ, Strouse PJ, Soskolne E, et al. Efficacy of recombinant human deoxyribonuclease I in the hospital management of respiratory syncytial virus bronchiolitis. Chest 2001;120:203-8.

12 Nenna R, Tromba V, Berardi R, et al. Recombinant human deoxyribonuclease treatment in hospital management of infants with moderate-severe bronchiolitis. Eur I Inflamm 2009;7:169-74.

13 Enriquez A, Chu IW, Mellis C et al. Nebulised deoxyribonuclease for viral bronchiolitis in children younger than 24 months. Cochrane Database Syst Rev 2012;11:CD008395

14 Merkus PJ, de Hoog M, van Gent R, et al. DNase treatment for atelectasis in infants with severe respiratory syncytial virus bronchiolitis. Eur Respir J 2001;18:734-7. 